Statistics in the Twenty-First Century: Special Volume

In Honour of Distinguished Professor Dr. Mir Masoom Ali

On the Occasion of his 75th Birthday Anniversary

PJSOR, Vol. 8, No. 3, pages 635-643, July 2012

\title{
Inference on Reliability in the Gamma and Inverted Gamma Distributions
}

Jungsoo Woo

Department of Statistics

Yeungnam University

Gyongsan, South Korea

jswoo@ynu.ac.kr

\begin{abstract}
We derive distributions of ratio for two independent gamma variables and two independent inverted gamma variables and then we observe the skewness of two ratio densities. We then consider inference on reliability in two independent gamma random variables and two independent inverted gamma random variables each having known shape parameters.
\end{abstract}

Keywords: Beta function, Hypergeometric function, Inverted gamma, Likelihood ratio test, Reliability, Whittaker function.

\section{Introduction}

For two independent random variables $X$ and $Y$ and a real number $c$, the probability $P(X<c Y)$ induces the following facts: (i) the probability $P(X<c Y)$ is the reliability when the real number $c$ equals one, (ii) the probability $P(X<c Y)$ is the distribution of the ratio $X /(X+Y)$ when $c=t /(1-t)$ for $0<t<1$, and (iii) the probability $P(X<c Y)$ is the density of a skewed random variable if $X$ and $Y$ are symmetric random variables about origin.

The reliability will increase the need for industry to perform systematic study for the identifications and reduction of causes of failures. These reliability studies must be performed by persons who (i) can identify and quantify the modes of failures, (ii) know how to obtain and analyze the statistics of failure occurrences, and (iii) can construct mathematical models of failure that depend on, for example, the parameters of material strength or design quality, fatigue or wear resistance, and the stochastic nature of the anticipated duty cycle (see Saunders (2007)).

Many authors have considered properties of gamma distribution (see Johnson et al (1994)). McCool (1991) considered the inference problem on reliability $P(X<Y)$ in the Weibull case. Ali and Woo (2005 a \& b)studied inference on reliability $P(Y<X)$ in power function and Levy distributions. Pal, Ali, and Woo (2005) studied estimation of testing $P(Y>X)$ in two parameter exponential distribution. Ali, Pal, and Woo (2010) studied the ratio of two independent exponentiated Pareto variables. Saunders (2007) 
introduced reliability, life testing and prediction of service lives for engineers and other scientists.

In this paper, we derive distributions of ratio for two independent gamma and two independent inverted gamma random variables and then we observe the skewness of the two ratio densities. We then consider inference on reliability in two independent gamma random variables and two independence inverted gamma random variables each having known shape parameter.

\section{Distribution of ratio}

In this Section we consider density of ratio of two independent gamma random variables each having the following density functions in (2.1) and density of the ratio of two independent inverted gamma random variables each having the following density functions in (2.4).

\subsection{Gamma distribution}

Let $X$ and $Y$ be independent gamma random variables having the following densities.

$$
\begin{aligned}
& f_{X}(x)=\frac{1}{\Gamma\left(\alpha_{1}\right) \beta_{1}^{\alpha_{1}}} x^{\alpha_{1}-1} e^{-x / \beta_{1}}, \text { if } 0<x<\infty \text { and, } \\
& f_{Y}(y)=\frac{1}{\Gamma\left(\alpha_{2}\right) \beta_{2}^{\alpha_{2}}} x^{\alpha_{2}-1} e^{-x / \beta_{2}}, \text { if } 0<y<\infty,
\end{aligned}
$$

where $\alpha_{i}$ 's and $\beta_{i}$ 's are positive.

Let $W=Y / X$. Then from the quotient density in Rohatgi $(1976, \mathrm{p} .141)$ and formula 3.381(4) in Gradshteyn and Ryzhik (1965, p.317), density of $W=Y / X$ is given by

$$
f_{W}(w)=\frac{\Gamma\left(\alpha_{1}+\alpha_{2}\right)}{\Gamma\left(\alpha_{1}\right) \Gamma\left(\alpha_{2}\right)}\left(\frac{\beta_{1}}{\beta_{2}}\right)^{\alpha_{2}} \cdot w^{\alpha_{1}-1}\left(1+\frac{\beta_{1}}{\beta_{2}}\right) \cdot w^{-\alpha_{1}-\alpha_{2}}, \text { if } 0<w<\infty .
$$

In fact, $\int_{0}^{\infty} f_{W}(w) d w=1$ from formula 2.19 in Oberhettinger (1974, p.15).

From the density (2.2) and formula 3.5 in Oberhettinger (1974, p.26), moment generating function (mgf) $M_{W}(t)$ of $W$ is given by

$$
M_{W}(t)=\frac{\Gamma\left(\alpha_{1}+\alpha_{2}\right)}{\Gamma\left(\alpha_{1}\right) \Gamma\left(\alpha_{2}\right)}\left(-\frac{\beta_{2}}{\beta_{1}} t\right)^{\frac{\alpha_{1}-1}{2}} e^{-\frac{\beta_{2}}{\beta_{1}} \cdot \frac{t}{2}} W_{\frac{1}{2}-\frac{\alpha_{1}}{2}-\alpha_{2}}\left(-\frac{\beta_{2}}{\beta_{1}} t\right),
$$

where $W_{a, b}(x)$ is the Whittaker function, see Abramowitz and Stegun (1970, p.505).

It guarantees the existence of the $k$ th moment of $W$, and hence from formula 3.194(3) in Gradshten and Ryzhik (1965, p.285), $k$ th moment of $W=Y / X$ is given by

$$
E\left(W^{k}\right)=\frac{\Gamma\left(\alpha_{1}-k\right) \Gamma\left(\alpha_{2}+k\right)}{\Gamma\left(\alpha_{1}\right) \Gamma\left(\alpha_{2}\right)}\left(\frac{\beta_{2}}{\beta_{1}}\right)^{k} \text {, if } \alpha_{1}>k=1,2,3, \ldots .
$$


We obtain the density of the ratio $R=X /(X+Y)=1 /(1+W)$ from (2.2) as the following.

Proposition 1: Let $X$ and $Y$ be independent random variables each having the respective density as in (2.1). Let $\rho=\frac{\beta_{2}}{\beta_{1}}$. Then the density of $R=X /(X+Y)$ is given by

$$
f_{R}(r)=\frac{\rho^{-\alpha_{2}}}{B\left(\alpha_{1}, \alpha_{2}\right)} r^{\alpha_{1}-1}(1-r)^{\alpha_{1}-1}(r+(1-r) / \rho)^{-\alpha_{1}-\alpha_{2}}, 0<r<1,
$$

where $B(a, b)$ is the beta function.

From Proposition 1 and formula 2.33 in Oberhettinger (1974, p.19), $k$ th moment of the ratio $R$ is obtained as follows. For $k=1,2,3, \ldots$ and $\rho=\frac{\beta_{2}}{\beta_{1}}$,

$$
E\left(R^{k}\right)=\left\{\begin{array}{l}
\frac{\Gamma\left(\alpha_{1}+\alpha_{2}\right) \Gamma\left(\alpha_{1}+k\right)}{\Gamma\left(\alpha_{1}+\alpha_{2}+k\right) \Gamma\left(\alpha_{2}\right)} \rho^{\alpha_{1}} \quad{ }_{2} F_{1}\left(\alpha_{1}+\alpha_{2}, \alpha_{1} ; \alpha_{1}+\alpha_{2}+k ; 1-\rho\right) \text { if } 0<\rho<1 \\
\frac{\Gamma\left(\alpha_{1}+\alpha_{2}\right) \Gamma\left(\alpha_{1}+k\right)}{\Gamma\left(\alpha_{1}+\alpha_{2}+k\right) \Gamma\left(\alpha_{2}\right)} \rho^{1-\alpha_{1}-2 \alpha_{2}-k} \quad{ }_{2} F_{1}\left(\alpha_{1}+\alpha_{2}, \alpha_{2} ; \alpha_{1}+\alpha_{2}+k ; 1-\frac{1}{\rho}\right) \text { if } 1<\rho
\end{array}\right.
$$

where ${ }_{2} F_{1}(a, b ; c ; x)$ is the hypergeometric function, see Abramowitz and Stegun (1970, p.555).

Table 1: Means, Variances, and Coefficients of Skewness for the density of ratio $R$

\begin{tabular}{|c|c|c|c|c|c|c|c|c|c|c|c|}
\hline$\alpha_{1}$ & $\alpha_{2}$ & $\rho$ & Mean & Variance & Skewness & $\alpha_{1}$ & $\alpha_{2}$ & $\rho$ & Mean & Variance & Skewness \\
\hline \multirow[t]{15}{*}{$1 / 3$} & \multirow[t]{5}{*}{$1 / 3$} & $1 / 4$ & .63134 & .14094 & -0.57650 & \multirow[t]{7}{*}{1} & \multirow[t]{2}{*}{1} & 2 & .38629 & .07819 & 0.48613 \\
\hline & & $1 / 2$ & .56611 & .14778 & -0.28375 & & & 4 & .28280 & .06483 & 1.00876 \\
\hline & & 1 & .5 & .125 & 0 & & \multirow[t]{5}{*}{3} & $1 / 6$ & .56630 & .06495 & -0.44687 \\
\hline & & 2 & .43389 & .14778 & 0.28375 & & & $1 / 4$ & .49175 & .06400 & -0.15461 \\
\hline & & 4 & .36866 & .14094 & 0.57650 & & & $1 / 2$ & .36447 & .05397 & 0.34031 \\
\hline & \multirow[t]{5}{*}{1} & $1 / 4$ & .41303 & .11923 & 0.25304 & & & 1 & .25 & .03750 & 0.34031 \\
\hline & & $1 / 2$ & .32870 & .10183 & 0.62912 & & & 2 & .15888 & .02146 & 1.44438 \\
\hline & & 1 & .25 & .08036 & 1.05830 & \multirow[t]{16}{*}{3} & \multirow[t]{5}{*}{$1 / 3$} & $1 / 4$ & .95655 & .01256 & -6.39987 \\
\hline & & 2 & .18244 & .05849 & 1.56897 & & & $1 / 2$ & .93255 & .01615 & -4.13837 \\
\hline & & 4 & .12575 & .03941 & 2.20195 & & & 1 & .89410 & .02545 & -2.48378 \\
\hline & \multirow[t]{5}{*}{3} & $1 / 4$ & .22862 & .06214 & 1.00151 & & & 2 & .83895 & .04180 & -1.58436 \\
\hline & & $1 / 2$ & .15637 & .03861 & 1.48985 & & & 4 & .76766 & .06414 & -1.01868 \\
\hline & & 1 & .1 & .02077 & 2.08167 & & \multirow[t]{6}{*}{1} & $1 / 4$ & .90573 & .01023 & -2.13336 \\
\hline & & 2 & .06001 & .00966 & 2.81950 & & & $1 / 2$ & .84112 & .02146 & -1.44441 \\
\hline & & 4 & .03408 & .00392 & 3.75406 & & & 1 & .75 & .03750 & -0.86067 \\
\hline \multirow[t]{8}{*}{1} & \multirow[t]{5}{*}{$1 / 3$} & $1 / 4$ & .86823 & .04388 & -2.2693 & & & 2 & .63553 & .05397 & -0.34031 \\
\hline & & $1 / 2$ & .81379 & .06152 & -1.57083 & & & 4 & .50825 & .06401 & 0.15461 \\
\hline & & 1 & .74621 & .08224 & -1.05286 & & & 6 & .43370 & .06495 & .044687 \\
\hline & & 2 & .66829 & .10285 & -0.62245 & & \multirow[t]{5}{*}{3} & $1 / 4$ & .76861 & .02089 & $-1,10089$ \\
\hline & & 4 & .58458 & .11964 & -0.2467 & & & $1 / 2$ & .64468 & .03116 & -0.53053 \\
\hline & \multirow[t]{3}{*}{1} & $1 / 4$ & .71720 & .06483 & -1.00876 & & & 1 & .5 & .04571 & 0 \\
\hline & & $1 / 2$ & .61371 & .07819 & -0.48613 & & & 2 & .35532 & .03116 & 0.53053 \\
\hline & & 1 & .5 & .08333 & 0 & & & 4 & .23139 & .02089 & 1.1089 \\
\hline
\end{tabular}
in the Gamma Distribution 
From $k$ th moment of the ratio $R$ in (2.3) and recursion formulas of hypergeometric function in Abramowitz and Stegun (1970, p.558), Table 1 provides numerical values of mean, variance, and coefficient of skewness for ratio density in Proposition 1.

From Table 1 we observe the following trends of the ratio density in Proposition 1.

Fact 1: Let $X$ and $Y$ be independent gamma random variables each having the density as given in (2.1). Then for $\rho=\beta_{2} / \beta_{1}$, and the ratio $R=X /(X+Y)$, we observe the following.

(i) When $\alpha_{1}=\alpha_{2}$, the density of the ratio $R$ is symmetric at $r=1 / 2$ when $\rho=1$, the density is left-skewed when $\rho<1$, it is right-skewed when $\rho>1$.

(ii) It is left-skewed when $\rho=1 / 4(2) 4$ for $\left(\alpha_{1}, \alpha_{2}\right)=(1,1 / 3)$ and $(3,1 / 3)$, but it is rightskewed when $\rho=1 / 4(2) 4$ for $\left(\alpha_{1}, \alpha_{2}\right)=(1 / 3,1)(1 / 3,3)$.

\subsection{Inverted gamma distribution}

We now consider the density of the ratio of two independent inverted gamma random variables $U$ and $V$, each having the following respective density. In the context of the gamma densities in (2.1), $U=1 / X$ and $V=1 / Y$.

$$
\begin{aligned}
& f_{U}(u)=\frac{1}{\Gamma\left(\alpha_{1}\right) \beta_{1}^{\alpha_{1}}} u^{-\alpha_{1}-1} e^{-u^{-1} / \beta_{1}}, u>0 \text { and }, \\
& f_{V}(v)=\frac{1}{\Gamma\left(\alpha_{2}\right) \beta_{2}^{\alpha_{2}}} v^{-\alpha_{2}-1} e^{-v^{-1} / \beta_{2}}, v>0 .
\end{aligned}
$$

Let $Q=V / U$. Then from Rohatgi (1976, p.141) and formula 3.381(4) in Gradshteyn and Ryzhik (1965, p.317), the density of $Q=V / U$ is obtained as

$$
f_{Q}(w)=\frac{\Gamma\left(\alpha_{1}+\alpha_{2}\right)}{\Gamma\left(\alpha_{1}\right) \Gamma\left(\alpha_{2}\right)}\left(\beta_{2} / \beta_{1}\right)^{\alpha_{1}} \cdot w^{-\alpha_{2}-1}\left(\beta_{2} / \beta_{1}+1 / w\right)^{-\alpha_{1}-\alpha_{2}}, 0<w<\infty
$$

In fact, $\int_{0}^{\infty} f_{Q}(w) d w=1$ by formula 2.19 in Oberhettinger $(1974, \mathrm{p} .15)$.

We obtain the density of $R_{1}=U /(U+V)=1 /(1+Q)$ from (2.5) as follows.

Proposition 2: Let $U$ and $V$ be independent inverted gamma random variables each having the respective density in (2.4). Then the density of the ratio $R_{1}$ is given by

$$
f_{R_{1}}(r)=\frac{\rho^{\alpha_{1}}}{B\left(\alpha_{1}, \alpha_{2}\right)} r^{-\alpha_{2}-1}(1-r)^{-\alpha_{1}-1}(r+\rho(1-r))^{-\alpha_{1}-\alpha_{2}}, 0<r<1,
$$

where $\rho=\beta_{2} / \beta_{1}$.

From Proposition 2 and formula 2.33 in Oberhettinger (1974, p.19), $k$ th moment of the ratio $R_{1}$ is given below. For $k=1,2,3, \ldots$ and $\rho=\beta_{2} / \beta_{1}$, 


$$
E\left(R_{1}^{k}\right)=\left\{\begin{array}{l}
\frac{\Gamma\left(\alpha_{1}+\alpha_{2}\right) \Gamma\left(\alpha_{1}+k\right)}{\Gamma\left(\alpha_{1}+\alpha_{2}+k\right) \Gamma\left(\alpha_{2}\right)} \rho^{2 \alpha_{1}+\alpha_{2}+k-1} \quad{ }_{2} F_{1}\left(\alpha_{1}+\alpha_{2}, \alpha_{1} ; \alpha_{1}+\alpha_{2}+k ; 1-\rho\right) \text { if } 0<\rho<1 \\
\frac{\Gamma\left(\alpha_{1}+\alpha_{2}\right) \Gamma\left(\alpha_{1}+k\right)}{\Gamma\left(\alpha_{1}+\alpha_{2}+k\right) \Gamma\left(\alpha_{2}\right)} \rho^{-\alpha_{2}} \quad{ }_{2} F_{1}\left(\alpha_{1}+\alpha_{2}, \alpha_{2} ; \alpha_{1}+\alpha_{2}+k ; 1-\frac{1}{\rho}\right) \text { if } 1<\rho .
\end{array}\right.
$$

Let us observe the following relation between $R_{1}$ and $R$.

The ratio $R_{1}=U /(U+V)=\frac{1 / X}{(1 / X+1 / Y)}=Y /(X+Y)=1-X /(X+Y)=1-R$. Hence, $E\left(R_{1}\right)=1-E(R), \operatorname{Var}\left(R_{1}\right)=\operatorname{Var}(R)$, and skewness of $R_{1}=$ $E\left[\left(R_{1}-E\left(R_{1}\right)\right)^{3} /\left[\operatorname{Var}\left(R_{1}\right)\right]^{3 / 2}=-E\left[(R-E(R))^{3} /[\operatorname{Var}(R)]^{3 / 2}=-\right.\right.$ skewness of $R$. Thus the mean, variance, and skewness of $R_{1}$ can be numerically obtained from Table 1 without providing a separate Table.

Proposition 3: Let $\mathrm{U}$ and $\mathrm{V}$ be two independent inverted gamma random variables each having the density respectively in (2.4). For ratio $R_{1}=U /(U+V)$ and $R=X /(X+Y)$, skewness for $R_{1}$ and $R$ are in the opposite direction, where $(X, Y)$ is a pair of independent gamma variables each having the respective density as in (2.1).

\section{Reliability $P(Y<X)$ in the Gamma Case}

In this Section we consider inference on reliability in two independent gamma random variables each having density with known respective shape parameter as in (2.1).

From the density (2.2) and formulas 3.381(1)\&(2) in Gradshteyn and Ryzhik (1965, p.317), we obtain the reliability as follows.

Proposition 4 Let $X$ and $Y$ be two independent gamma random variables each having the respective density as in (2.1) with known shape parameter. Let $\rho=\beta_{2} / \beta_{1}$. Then

$$
R(\rho)=P(Y<X)=\frac{\Gamma\left(\alpha_{1}+\alpha_{2}\right)}{\Gamma\left(\alpha_{1}\right) \Gamma\left(\alpha_{2}\right)} \cdot \frac{\rho^{\alpha_{1}}}{(1+\rho)^{\alpha_{1}+\alpha_{2}}} \quad{ }_{2} F_{1}\left(1, \alpha_{1}+\alpha_{2} ; 1+\alpha_{2} ; \frac{1}{1+\rho}\right) .
$$

From Proposition 4 and formula 15.2.1 in Abramowitz and Stegun (1970, p.557), we obtain the following.

Proposition 5: Let $X$ and $Y$ be two independent gamma random variables each having the respective density as in (2.1). If mean of $Y$ is greater than that of $X$, i.e., if $\alpha_{2} \beta_{2}>\alpha_{1} \beta_{1}$ in densities (2.1), then reliability $R(\rho)=P(Y<X)$ is a monotone decreasing finction of $\rho$. If mean of $X$ is greater than mean of $Y$, i.e., if $\alpha_{2} \beta_{2}<\alpha_{1} \beta_{1}$ in densities (2.1), then reliability $R(\rho)=P(Y<X)=1-P(X<Y)$ is a monotone increasing function of $\rho$. 
We now consider inference on reliability $P(Y<X)$ when the shape parameters $\alpha_{1}$ and $\alpha_{2}$ are known. Because $R(\rho)$ is a monotonic function of $\rho$, inference on reliability is equivalent to inference on $\rho$ (see $\mathrm{McCool}$ (1991)). Hence, it is sufficient for us to consider inference on $\rho \equiv \beta_{2} / \beta_{1}$ when the shape parameters $\alpha_{1}$ and $\alpha_{2}$ are known.

We get the following Lemma easily from formulas 3.381(4) in Gradshteyn and Ryzhik (1965, p.317).

Lemma 1: Let $X$ be a gamma random variable having mean $\alpha \beta$ and variance $\alpha \beta^{2}$. Then

(a) $E(1 / X)=\frac{1}{(\alpha-1) \beta}$, if $\alpha>1$.

(b) $E\left(1 / X^{2}\right)=\frac{1}{(\alpha-1)(\alpha-2) \beta^{2}}$, if $\alpha>2$.

Assume $X_{1}, X_{2}, \ldots, X_{m}$ and $Y_{1}, Y_{2}, \ldots, Y_{n}$ be two independent samples from each density in (2.1) with known shape parameter, respectively. Then MLE $\hat{\beta}_{i}$ of $\beta_{i}, i=1,2$ are given by

$$
\hat{\beta}_{1}=\frac{1}{m \alpha_{1}} \sum_{i=1}^{m} X_{i} \text {, and } \hat{\beta}_{2}=\frac{1}{n \alpha_{2}} \sum_{i=1}^{n} Y_{i}
$$

Therefore, MLE $\hat{\rho}$ of $\rho$ is $\hat{\rho}=\hat{\beta}_{2} / \hat{\beta}_{1}$.

From Lemma 1, mean and variance of $\hat{\rho}$ are given by

$$
E(\hat{\rho})=\frac{m \alpha_{1}}{m \alpha_{1}-1} \cdot \rho,
$$

and

$$
\operatorname{Var}(\hat{\rho})=\frac{m^{2} \alpha_{1}^{2}\left(m \alpha_{1}+n \alpha_{2}-1\right)}{\left(m \alpha_{1}-1\right)^{2} n \alpha_{2}\left(m \alpha_{1}-2\right)} \cdot \rho^{2}, \text { if } m \alpha_{1}>2
$$

From expectation in (3.1), an unbiased estimator $\tilde{\rho}$ of $\rho$ is defined by

$$
\tilde{\rho}=\frac{m \alpha_{1}-1}{n \alpha_{2}} \cdot \frac{\sum_{j=1}^{n} Y_{j}}{\sum_{i=1}^{m} X_{i}} .
$$

From Lemma 1, variance of $\tilde{\rho}$ is given by

$$
\operatorname{Var}(\tilde{\rho})=\frac{m \alpha_{1}+n \alpha_{2}-1}{n \alpha_{2}\left(m \alpha_{1}-2\right)} \cdot \rho^{2}
$$


From the results in (3.1), (3.2), and (3.3), we find that $\operatorname{MSE}(\tilde{\rho})<\operatorname{MSE}(\hat{\rho})$ and then we obtain the following from equivalence of inference between $\rho$ and $R(\rho)$ (see McCool (1991)).

Proposition 6: Assume $X_{1}, X_{2}, \ldots, X_{m}$ and $Y_{1}, Y_{2}, \ldots, Y_{n}$ be two independent random samples from the respective densities in (2.1) with known shape parameters. Then an estimator $R(\tilde{\rho})$ performs better than the MLE $R(\hat{\rho})$ in the sense of MSE if $m \alpha_{1}>2$.

\subsection{The shape parameter is a positive integer}

We consider interval estimator of $\rho$, especially if the shape parameters $\alpha_{i}$ 's in the densities (2.1) are known positive integers., i.e., the densities (2.1) belong to Erlang distributions. Then $\rho \cdot \hat{\beta}_{1} / \hat{\beta}_{2}$ is a pivot quantity having the $F$-distribution with degrees of freedom $\left(2 m \alpha_{1}, 2 n \alpha_{2}\right)$. Therefore, an $(1-\gamma) 100 \%$ confidence interval for $\rho$ is

$$
\left(\frac{1}{F_{\gamma / 2}\left(2 n \alpha_{2}, 2 m \alpha_{1}\right)} \cdot \frac{\hat{\beta}_{2}}{\hat{\beta}_{1}}, \quad F_{\gamma / 2}\left(2 m \alpha_{1}, 2 n \alpha_{2}\right) \cdot \frac{\hat{\beta}_{2}}{\hat{\beta}_{1}}\right) \text {, }
$$

where $\int_{c}^{\infty} h(t) d t=\gamma / 2, c \equiv F_{\gamma / 2}\left(2 m \alpha_{2}, 2 n \alpha_{2}\right), h(t)$ is the density of $F$-distribution with degrees of freedom $\left(2 m \alpha_{1}, 2 n \alpha_{2}\right)$.

Next, we consider the testing of the following hypothesis:

$$
H_{0}: \beta_{1}=\beta_{2}=\beta(\rho=1) \text { against } H_{1}: \beta_{1} \neq \beta_{2}(\rho \neq 1) \text {. }
$$

Applying the likelihood ratio test, we reject $H_{0}$ if and only if

$$
\lambda\left(x_{1}, x_{2}, \ldots, x_{m} ; y_{1}, y_{2}, \ldots, y_{n}\right)=\frac{f_{X}\left(x_{1}, x_{2}, \ldots, x_{m} ; \hat{\beta}\right) \cdot f_{Y}\left(y_{1}, y_{2}, \ldots, y_{n} ; \hat{\beta}\right)}{f_{X}\left(x_{1}, x_{2}, \ldots, x_{m} ; \hat{\beta}_{1}\right) \cdot f_{Y}\left(y_{1}, y_{2}, \ldots, y_{n} ; \hat{\beta}_{2}\right)} \leq c
$$

where $c$ is a constant and $\hat{\beta}=\frac{1}{m \alpha_{1}+n \alpha_{2}}\left(\sum_{i=1}^{m} X_{i}+\sum_{j=1}^{n} Y_{j}\right)$.

The above likelihood ratio results in the following equvalent test:

Reject $H_{0}$ if for a given test size $\gamma$, where $0<\gamma<1$,

$$
\frac{\hat{\beta}_{2}}{\hat{\beta}_{1}}<\frac{1}{F_{\gamma / 2}\left(2 m \alpha_{1}, 2 n \alpha_{2}\right)} \text { or } \frac{\hat{\beta}_{2}}{\hat{\beta}_{1}}>F_{\gamma / 2}\left(2 n \alpha_{2}, 2 m \alpha_{1}\right) \text {. }
$$

\subsection{The shape parameter is known positive}

We consider an asymptotic interval estimate for $\rho$ if the shape parameters $\alpha_{1}$ and $\alpha_{2}$ are known positve. Then using the asymptotic property of MLE and mean and variance in (3.1) and (3.2) of $\hat{\rho}$, for large $m$ and $n$,

$$
\frac{\hat{\rho}-\rho}{\rho \sqrt{m^{2} \alpha_{1}^{2}\left(m \alpha_{1}+n \alpha_{2}-1\right) /\left(\left(m \alpha_{1}-1\right)^{2} n \alpha_{2}\left(n \alpha_{1}-2\right)\right)}}
$$

has an asymptotic standard normal distribution. 
From (3.1) and (3.2) we find that $\hat{\rho}$ is a consistent estimator of $\rho$ and then from the limit Theorem in Rohatgi (1976, p.253), we obtain the following. For large $m$ and $n$,

$$
\frac{\hat{\rho}-\rho}{\hat{\rho} \sqrt{m^{2} \alpha_{1}^{2}\left(m \alpha_{1}+n \alpha_{2}-1\right) /\left(\left(m \alpha_{1}-1\right)^{2} n \alpha_{2}\left(n \alpha_{1}-2\right)\right)}}
$$

has an asymptotic standard normal distribution.

Therefore, an asymptotic $(1-\gamma) 100 \%$ confidence interval for $\rho$ is given by

$$
\hat{\rho} \cdot\left(1 \mp z_{\gamma / 2} \cdot \sqrt{m^{2} \alpha_{1}^{2}\left(m \alpha_{1}+n \alpha_{2}-1\right) /\left(\left(m \alpha_{1}-1\right)^{2} n \alpha_{2}\left(n \alpha_{1}-2\right)\right)}\right),
$$

where $\gamma / 2=\int_{z_{\gamma / 2}}^{\infty} \phi(t) d t$, where $\phi(t)$ is the standard normal density.

We also wish to test the following

$$
H_{0}: \beta_{1}=\beta_{2}=\beta(\rho=1) \text { against } H_{1}: \beta_{1} \neq \beta_{2}(\rho \neq 1)
$$

For large $m$ and $n$ and given a test size $\gamma, 0<\gamma<1$, reject $H_{0}$ if

$$
\begin{aligned}
& \frac{\hat{\beta}_{2} / \hat{\beta}_{1}-1}{\hat{\beta}_{2} / \hat{\beta}_{1} \sqrt{m^{2} \alpha_{1}^{2}\left(m \alpha_{1}+n \alpha_{2}-1\right) /\left(\left(m \alpha_{1}-1\right)^{2} n \alpha_{2}\left(n \alpha_{1}-2\right)\right)}}<-z_{\gamma / 2} \text { or } \\
& \frac{\hat{\beta}_{2} / \hat{\beta}_{1}-1}{\hat{\beta}_{2} / \hat{\beta}_{1} \sqrt{m^{2} \alpha_{1}^{2}\left(m \alpha_{1}+n \alpha_{2}-1\right) /\left(\left(m \alpha_{1}-1\right)^{2} n \alpha_{2}\left(n \alpha_{1}-2\right)\right)}}>z_{\gamma / 2}
\end{aligned}
$$

\section{Reliability $\mathbf{P}(V<U)$ in the inverted gamma case}

In this Section we consider inference on reliability in two independent inverted gamma random variables each having respective density as in (2.4). Using the same method for the gamma case, we obtain the following.

Proposition 7: Let $U$ and $V$ be independent inverted gamma random variables each having the respective density in (2.4) with known shape parameters. Then for $\rho=\beta_{2} / \beta_{1}$,

$$
\begin{aligned}
& R(\rho)=P(V<U)=1-P(1 / V<1 / U) \\
& \quad=1-\frac{\Gamma\left(\alpha_{1}+\alpha_{2}\right)}{\Gamma\left(\alpha_{1}\right) \Gamma\left(\alpha_{2}\right)} \frac{\rho^{\alpha_{1}}}{(1+\rho)^{\alpha_{1}+\alpha_{2}}} \quad{ }_{2} F_{1}\left(1, \alpha_{1}+\alpha_{2} ; 1+\alpha_{2} ; \frac{1}{1+\rho}\right)
\end{aligned}
$$

is a monotone function of $\rho$. The proof comes from Propositions 4 and 5.

Assume $U_{1}, U_{2}, \ldots, U_{m}$ and $V_{1}, V_{2}, \ldots, V_{n}$ be two independent random samples from each respective density in (2.4) with known shape parameters. Then $1 / U_{1}, 1 / U_{2}, \ldots, 1 / U_{m}$ and $1 / V_{1}, 1 / V_{2}, \ldots, 1 / V_{n}$ are two independent random samples from the respective densities in (2.1) with known shape parameters. 
The MLE $\hat{\beta}_{i}$, of $\beta_{i}, i=1,2$ are given by

$$
\hat{\beta}_{1}=\frac{1}{m \alpha_{1}} \sum_{i=1}^{m} \frac{1}{U_{i}} \text { and } \hat{\beta}_{2}=\frac{1}{n \alpha_{2}} \sum_{j=1}^{n} \frac{1}{V_{j}} \text {. }
$$

Using similar arguments as in Section 3, we can consider inference on reliability $R(\rho)$ in the inverted gamma distribution using the two random samples $1 / U_{1}, 1 / U_{2}, \ldots, 1 / U_{m}$ and $1 / V_{1}, 1 / V_{2}, \ldots, 1 / V_{n}$.

\section{References}

1. Abramowitz, M. and Stegun, I. A. (1970). Handbook of Mathematical Functions. Dover Publications Inc., New York.

2. Ali, M. Masoom, Pal, M., and Woo, J. (2010). On the ratio of two independent exponentiated Pareto variables. Austrian Journal of Statistics 39, 329-340.

3. Ali, M. Masoom, Pal, M., and Woo. J. (2005a). Inferences on reliability $\mathrm{P}(Y<X)$ in a power function distribution. Journal of Statistics \& Management Systems 8, 681-686.

4. Ali, M. Masoom, Pal, M., and Woo. J. (2005b). Inference on reliability $\mathrm{P}(Y<X)$ in the Levy distribution. Mathematics and Computer Modelling 41, 965-971.

5. Gradshteyn, I. S. and Ryzhik, I. M. (1965). Tables of Integrals, Series, and Products. Academic Press, New York.

6. Johnson, N. L., Kotz, S. and Balakrishnan, N. (1994). Continuous Univariate Distributions. Houghton Mifflin Com., Boston.

7. McCool, J. I. (1991). Inference on $\mathrm{P}(Y<X)$ in the Weibull case. Communications in Statistics - Simulation \& Computation 20, 129-148.

8. Oberhettinger, F. (1974). Tables of Mellin Transform. Springer-Verlag, New York, New York.

9. Pal, M., Ali, M. Masoom, and Woo, J. (2005). Estimation and testing of $\mathrm{P}(Y>X)$ in two parameter exponential distributions. Statistics 39, 415-428.

10. Rohatgi, V. K. (1976). An Introduction to Probability Theory and Mathematical Statistics. John Wiley \& Sons, New York.

11. Saunders, S. C. (2007). Reliability, Life Testing, and Prediction of Service Lives. Springer, New York. 